\title{
PERFORMANCE ANALYSIS OF SEVERAL SCHEDULING ALGORITHMS IN NAKAGAMI-M FADING CHANNELS
}

\author{
Hakkı Soy \\ Department of Electrical and Electronics Engineering, \\ Necmettin Erbakan University, Konya, Turkey
}

\begin{abstract}
This study investigates the performances of several scheduling algorithms (Round Robin, maximum SNR and opportunistic Round Robin) in the Medium Access Control (MAC) layer of wireless network with cellular architecture. The spectral efficiency and fairness are two important metrics to evaluate the performance of wireless systems. The Nakagami-m distribution is a generalized way to model the small scale fading and it can characterize the various wireless fading channel conditions by changing the value of $m$ parameter. The channel is assumed to have a Nakagami-m slow fading model and the opportunistic beamforming scheme is used to enlarge the rate / dynamic range of channel fluctuations through the employment of multiple antennas at the base station. The MATLAB based Monte Carlo simulations are implemented to show the effects of the antenna number and m parameter of the Nakagami distribution. The obtained results proved that the pure opportunistic max-SNR algorithm has a spectral efficiency advantage over the others, even though it has a bad fairness characteristic in high number of users. The main contribution of this study is showing that the effects of the Nakagami-m fading parameter and opportunistic beamforming on the performance of the well-known scheduling algorithms.
\end{abstract}

\section{KEYWORDS}

Spectral Efficiency, Fairness, Scheduling, Opportunistic Beamforming, Nakagami-m Fading

\section{INTRODUCTION}

Wireless networks have been one of the fastest growing segments in the telecommunications industry, ever since the first one was introduced in 1970. Recently, the fast evolution of wireless technologies has enabled the high-speed data services and the demand for higher data rates is increasing exponentially with each passing day [1]. Today, the most important challenge for wireless systems is the limited availability of the radio frequency spectrum. Therefore, the spectral efficiency is a primary consideration in the design of future wireless communications systems. Over the last decades, many researchers have looked at various methods to improve the spectral efficiency of wireless systems [2,3].

There are two types of wireless network architectures, cellular and ad hoc. The cellular architecture has evolved from mobile telephone systems and it has several potential advantages for future wireless networks because of its ability to operate using small transmission powers and David C. Wyld et al. (Eds) : NeCoM, SEAS, SP, CMCA - 2018 pp. 23-32, 2018. (C) CS \& IT-CSCP 2018

DOI : $10.5121 /$ csit.2018.80902 
to increase the spatial reuse in the network. Besides, the ad hoc network is a non-infrastructure architecture, in which users communicate with each other through several hops of short distance transmissions. The ad hoc model is specifically designed to support the military and emergency operations $[4,5]$.

The cellular networks are organized with a base station (BS) which is responsible for controlling the access to the wireless channel and forwarding data to the intended user. The BS provides the connectivity among the users to efficiently and fairly dispense the available bandwidth [6]. The scheduling is a method that enables multiple users to share the system resources (time-slots, transmit power, bandwidth, etc.) [7]. In BS, the scheduler makes a decision on which users it is going to serve during the next transmission period. The scheduling algorithm is said to be opportunistic when it takes the channel quality into consideration before it takes a scheduling decision. High spectral efficiency of a wireless cellular network can be achieved by allocating the system resources to the users opportunistically [8-10].

The key characteristic of the wireless channel is the fading due to the multipath propagation of the transmitted signal, which creates replicas of the transmitted signal that arrive at the receiver with different delays [11]. Due to the fading effect, the channel conditions of users have timevarying behaviour at a certain time and each user has different channel statistics. Therefore, different users experience different channel gains and this effect is called as multiuser diversity [12]. The utilization of multiuser diversity in a wireless cellular network can increase the information theoretic capacity by allowing opportunistic usage of the channel [13].

The statistical description of the radio channels is essential for wireless communication systems. There are several existing statistical channel models are used to in the literature describe the statistics of the multipath signals [14]. Rayleigh fading channel is an excellent approximation in a large number of practical scenarios when there is non-line of sight (NLOS) component present in received signal. Rician fading is suitable for the communication environment with LOS component. Beyond that, Nakagami-m fading offers more versatile channel model due to advantage of greater flexibility and accuracy in matching some experimental data than the Rayleigh and Rician channel models [15].

The multiuser diversity gains depend on the tail of the fading distribution and increases with the dynamic range of the channel fluctuations. But, in little scattering and/or slow fading environments, the dynamic range of channel fluctuations remains very small. In these cases, a practical solution can be provided with the employment of multiple antennas at the BS to induce large and fast channel fluctuations artificially in the downlink channel. Such a beamforming scheme is called opportunistic beamforming since the BS randomly varies the amplitude and phase values of transmitted signals from the antennas at each time slot. So, the multiuser diversity can still be exploited in cellular wireless networks [16].

This paper focus on the spectral efficiency of wireless links and the fairness of channel access in a cellular network by considering Nakagami-m fading as a model for the channel gains of users. The spectral efficiency is defined as the average transmitted data rate per unit bandwidth for a specified average transmit power and bit error rate (BER). The fairness refers to the equal allocation of network resources among the various users. The aim is demonstration of the effect of the different fading conditions over the spectral efficiency and fairness performances. The MATLAB based Monte Carlo simulations are implemented to analyse the performance of investigated scheduling algorithms. 


\section{SYSTEM MODEL}

The system of interest is a single cell of the cellular wireless network that consists of multiple users. The BS has access to the downlink channel, while uplink channel is shared among users. The downlink system model of the considered network setup is shown in Figure 1. The BS is equipped with $M$ antennas and serving $K$ users whereas each user is equipped with single antenna. The BS coordinates all data transmissions within its coverage range. The channels between the transmit antennas and the users experience fading paths which are modelled as i.i.d. Nakagami-m fading channels. The channel between the $k$ th user and the BS is denoted by $M \times 1$ vector

$\mathbf{h}_{\mathrm{k}}=\left[\begin{array}{llll}h_{\mathrm{k}, 1} & h_{\mathrm{k}, 2} & \ldots & h_{\mathrm{k}, \mathrm{M}}\end{array}\right]^{\mathrm{T}}$

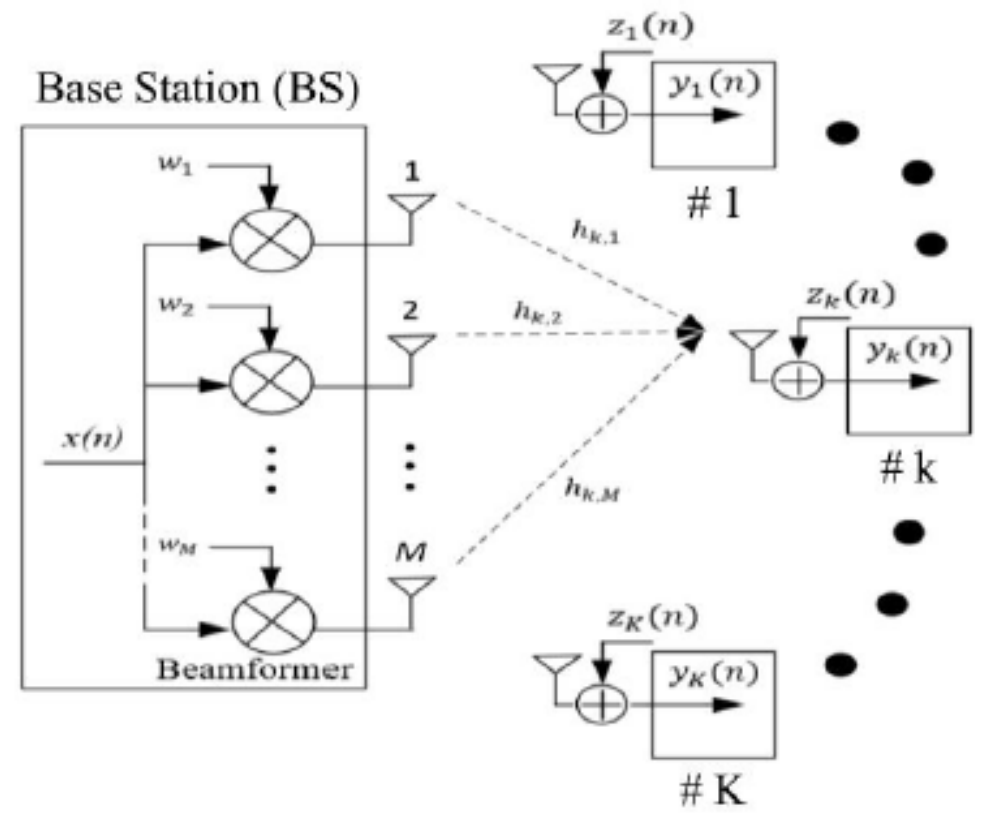

Figure 1. The system model of considered cellular network

The BS forms the beam by choosing the $M \times 1$ random beamforming vector $\mathbf{w}$ whose distribution is identical to the distribution of $\mathbf{h}_{\mathrm{k}}$ but normalized to keep the transmit power fixed, $\mathbf{w} \sim \mathbf{h} /\|\mathbf{h}\|$. The pilot signal $x(n)$ with power $\mathrm{E}\left[x^{2}(n)\right]=\mathrm{P}_{\mathrm{t}}$ is transmitted from the BS to the users. The received signal $y_{k}(\mathrm{n})$ at the kth user may be written as

$$
y_{k}(n)=\sqrt{P_{t}}\left(\mathbf{w}^{\mathrm{H}} \mathbf{h}_{k}\right) x(n)+z_{k}(n)
$$

where $z_{k}(n)$ is the circularly symmetric, complex, additive white Gaussian noise (AWGN) with distribution $\left(0, \sigma^{2}\right)$. Note that, by randomly changing the beamforming vector $\mathbf{w}$ at each time slot, the observed composite channel process of the kth user $\left(\mathbf{w}^{\mathrm{H}} \mathbf{h}_{\mathrm{k}}\right)$ changes from time-slot to timeslot due to the time-varying beamforming vector.

In order to simplify the analysis, we assume that the channel statistics of all the users are the same and the ratio of the transmit power to the noise variance $\left(\mathrm{P}_{t} / \sigma^{2}\right)$ is 1 . So, without loss of generality, the path loss together with all the other powers is lumped into the channel process. 
The quality of wireless links is usually measured by using signal-to-noise ratio (SNR) metric. Thus, with these assumptions, the SNR of the kth user can be written as

$$
\gamma_{k}=\mathbf{w}^{\mathrm{H}} \mathbf{h}_{k} \mathbf{h}_{k}^{\mathrm{H}} \mathbf{w}
$$

The probability density function (P.D.F.) of the instantaneous SNR is given as [15]:

$$
f_{\gamma}(\gamma)=\left(\frac{m}{\bar{\gamma}}\right)^{m} \frac{\gamma^{m-1}}{\Gamma(m)} e^{-m \frac{\gamma}{\gamma}}
$$

where $\bar{\gamma}$ is the average SNR of the received signal, m called as Nakagami parameter and $\Gamma($. denotes the Gamma function given by $\Gamma(\alpha)=\int_{0}^{\infty} t^{\alpha-1} e^{-t} d t$ [17]. The phase of the Nakagami fading is uniformly distributed over $[0,2 \pi]$. Then, the corresponding cumulative distribution function (C.D.F.) is expressed as [15]:

$$
F_{\gamma}(\gamma)=1-\frac{\Gamma\left(m, \frac{m}{\gamma} \gamma\right)}{\Gamma(m)}
$$

where $\Gamma(.,$.$) denotes the lower incomplete gamma function as \Gamma(\alpha, \beta)=\int_{0}^{\beta} t^{\alpha-1} e^{-t} d t$ [17].

The Nakagami-m distribution is a generalized distribution that can be used to model fading environments by changing the value of $m$ parameter. Rayleigh P.D.F. is obtained for $m=1$ as a special case, one-sided Gaussian P.D.F. is described by $m=1 / 2$ and the non-fading situation corresponds to $m \rightarrow \infty$. The Nakagami fading model is also able to cover severe $(1 / 2 \leq m \leq 1)$ and weak $(m>1)$ channel conditions than Rayleigh fading model [15].

\section{SCHEDULING ALgORITHMS}

In cellular wireless networks, the channel allocation task is organized by a scheduler runs on the BS. The users send request to BS and the requests are processed according to medium access control (MAC) protocol. Clearly, the MAC protocol determines the channel access strategy with predefined scheduling algorithm [19]. Behind the fading channel, another important problem, the spectral efficiency of the wireless channel is also influenced by the packet collision due to the simultaneous transmissions of multiple users. In order to provide a collision-free scheduling scheme, it is assumed that only a single node can use the channel at a certain time-slot. The most of the existing MAC protocols use the time division multiple access (TDMA) mechanism where the time axis is divided into equal-sized time-slots. The scheduling algorithm selects a single user at a certain time-slot $[20,21]$.

In general, scheduling algorithms can be classified as opportunistic, semi-opportunistic and nonopportunistic [22]. Opportunistic algorithms maximize the spectral efficiency at the expense of 
full feedback from all the users. The BS requires the channel state information (CSI) from all users to make a scheduling decision on which users it is going to serve during the next time-slot. The downlink channels can be easily estimated by users via pilot signal broadcast, but uplink channels need feedback transmissions from all users. Based on the collected CSI through feedback channel, the BS makes notifies the all users about the assigned user which access the channel alone. But, the excessive feedback overhead increases the delay which degrades the performance of bandwidth-intensive and time-critical applications. Unlike, the non-opportunistic algorithms simply assign the time-slots to users as independent from their instantaneous channel conditions. This type strategy does not require CSI feedback, but it does not offer multiuser diversity gain with spectral efficiency advantage. Between them, the semi-opportunistic algorithms remove the feedback overhead with a slight loss in the spectral efficiency [23].

This study focuses on these three scheduling algorithms which have different characteristics each other. In literature, these scheduling algorithms are named as Round Robin (RR), maximum SNR (max-SNR) and opportunistic Round Robin (ORR). In non-opportunistic RR algorithm, the timeslots are allocated to the users in a sequential fashion regardless of their channel conditions. The max-SNR algorithm always selects the user with the best channel quality in an opportunistic approach [24]. Finally, in ORR algorithm, the best user among all is chosen for the first time-slot. At the next time-slot, this user taken out of the competition and the best out of the remaining users is selected for channel assignment. This procedure is repeated until the last time-slot in a round, where the latter user is scheduled. The ORR algorithm ensures the constraint that the $K$ number of users should get exactly one time-slot each within the same round as well as the RR algorithm $[25,26]$.

The spectral efficiency and fairness of wireless cellular networks are depending on the selected scheduling algorithm in MAC layer. The spectral efficiency is a function of the average SNR of users that access to the channel and the fairness is related with the priority of the scheduler. In RR algorithm, the P.D.F. of assigned users is given by equation (3). So, the spectral efficiency $\Psi_{\mathrm{RR}}$ is expressed as follows:

$$
\Psi_{\mathrm{RR}}=\int_{0}^{\infty} \log _{2}(1+\gamma) f_{\gamma}(\gamma) d \gamma=\left(\frac{m}{\bar{\gamma}}\right)^{m} \frac{1}{\Gamma(m)} \int_{0}^{\infty} \log _{2}(1+\gamma) \gamma^{m-1} e^{-m \frac{\gamma}{\bar{\gamma}}} d \gamma .
$$

In max-SNR algorithm, the spectral efficiency $\Psi_{\max -\mathrm{SNR}}$ can be formulated using a stochastic framework by using the P.D.F. and C.D.F. expressions in equation (3) and (4), respectively:

$$
\Psi_{\max -\mathrm{SNR}}=\int_{0}^{\infty} \log _{2}(1+\gamma) f_{\dot{\gamma}^{*}}(\gamma) d \gamma=K \int_{0}^{\infty} \log _{2}(1+\gamma) F_{\gamma}^{K-1}(\gamma) f_{\gamma}(\gamma) d \gamma \text {. }
$$

In this formulation, the $f_{\gamma} \cdot(\gamma)$ term denotes the SNR of selected user with maximum channel quality among all users.

On the other hand, in the ORR algorithm, the spectral efficiency $\Psi_{\text {ORR }}$ has a more complex formulation due to its iterative form as expressed below: 
$\Psi_{\mathrm{ORR}}=\int_{0}^{\infty} \log _{2}(1+\gamma) f_{\gamma^{*}}(\gamma) d \gamma=\frac{1}{K} \int_{0}^{\infty} \log _{2}(1+\gamma) \sum_{k=1}^{K} k F_{\gamma}^{k-1}(\gamma) f_{\gamma}(\gamma) d \gamma$

In this formulation, the $f_{\gamma^{\prime}}(\gamma)$ term denotes the SNR of selected user with maximum channel quality among users in still competition with others. The complicated form of the spectral efficiency formulations in equation (5), (6) and (7) makes it difficult to find a closed form solution. These expressions can be calculated numerically and the details of the closed form solutions are beyond the scope of this paper.

Finally, the fairness is defined by how equally the channel assignments are allocated to users. The Jain's fairness index (JFI) is often used to measure the fairness of scheduling algorithms in cellular wireless networks [27]. In case of the time-slot based resource allocation, the JFI is defined as follows:

$$
\mathrm{I}_{\mathrm{JF}}=\frac{\left|\sum_{k=1}^{K} p_{k}\right|^{2}}{K \sum_{k=1}^{K} p_{k}^{2}}
$$

where $p_{\mathrm{k}}$ is the number of assigned time-slots to user $k$ in a round [28].

\section{SimUlation RESULTS}

In this study, the performance of mentioned scheduling algorithms is investigated in terms of spectral efficiency and fairness over generalized Nakagami-m fading channel. The MATLAB software is used to present the statistical (Monte Carlo) simulation results of investigated scheduling algorithm's performances. The obtained results are shown for both $2 \times 1$ and $4 \times 1$ antenna configurations over Nakagami-0.6, Nakagami-1 and Nakagami-3 cases separately. In Monte Carlo simulations, the number of iterations is chosen as 1000 to achieve an acceptable convergence and the number of time slots in an iteration is taken as the number of users in the network. It is assumed that the channel is frequency flat, block fading and the channel vector $\mathbf{h}_{k}$ remains constant during a fixed number of time-slots called one frame and changes between different frames independently. The average SNR is assumed to be the same for all users, while the instantaneous SNR values of users depend on the channel fading realization in each frame.

Figure 2 shows the spectral efficiency performance of scheduling algorithms in $2 \times 1$ antenna configuration. The max-SNR algorithm has the best spectral efficiency, while the RR algorithm has the worst. In max-SNR algorithm, the spectral efficiency increases for larger numbers of users. It is meaning that there exists a favorable user whose instantaneous channel vector matches the beamforming vector and who can maximize the received SNR. Note that, in max-SNR algorithm, when the value of $m$ parameter increases, the spectral efficiency performance decreases due to the insufficient multiuser diversity gain. But, the spectral efficiency performance increases with the increasing $m$ values in RR and ORR algorithms. Because, the LOS component 
plays dominant role for $m>1$ and it is possible to get better signal from the BS. The spectral efficiency of RR algorithm is independent from the number of users.

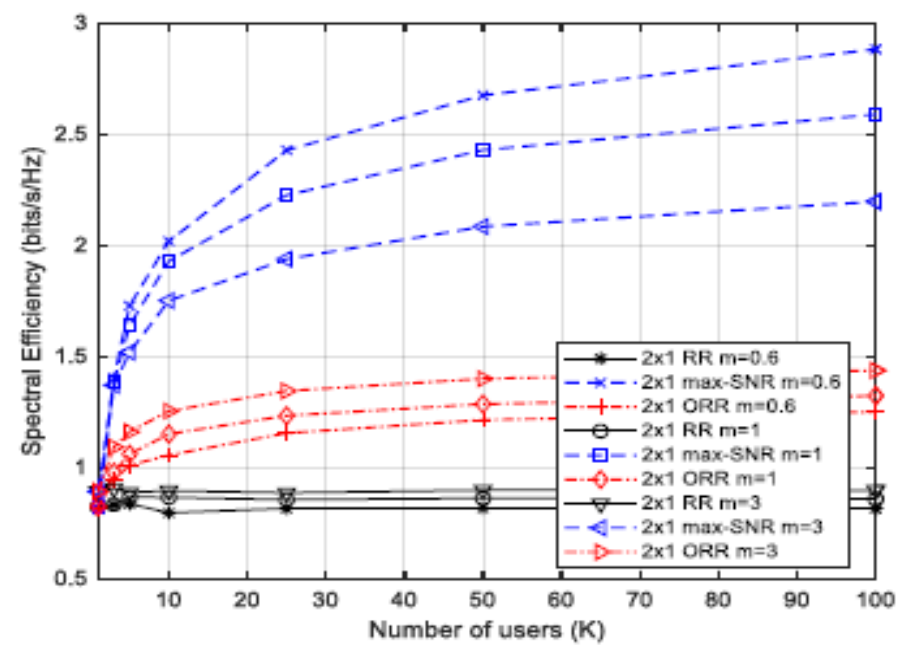

Figure 2. Spectral efficiency of scheduling algorithms in $2 \times 1$ antenna configuration for different $\mathrm{m}$ values

The fairness of scheduling algorithms in $2 \times 1$ antenna configuration is shown by Figure 3 for different $m$ values. It means that the higher value of JFI implies higher fairness in channel assignment. The RR and ORR algorithms have the best fairness $(\mathrm{JFI}=1)$ and the max-SNR algorithm has the worst fairness performance. It is clearly shown that the fairness performance of the max-SNR algorithm decreases with the increasing user numbers. Note also that, the increasing the value of $m$ parameter makes a positive effect and slightly improves the fairness index of pure opportunistic max-SNR algorithm. According that, the max-SNR algorithm provides better channel assignment in weaker channel conditions than Rayleigh model.

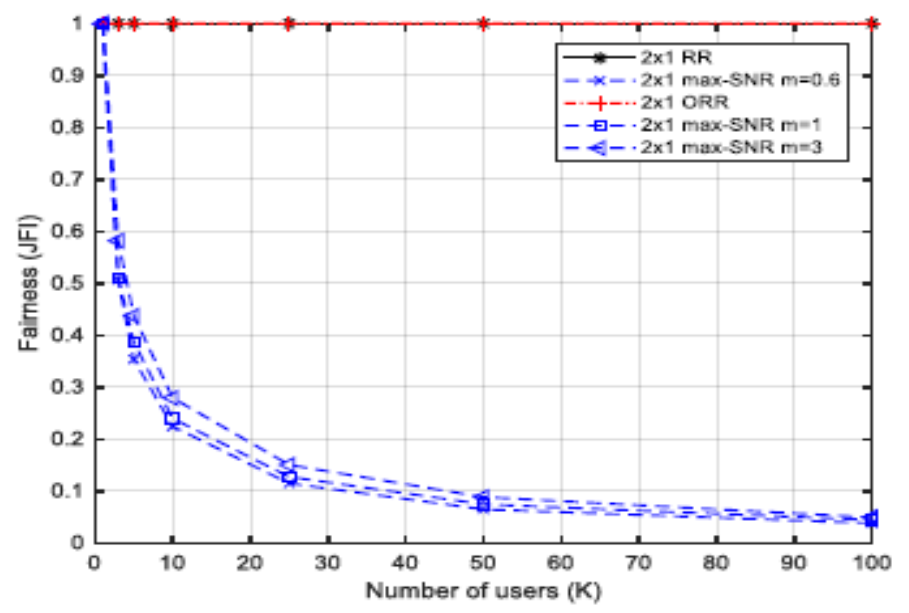

Figure 3. Fairness of scheduling algorithms in $2 \times 1$ antenna configuration for different $\mathrm{m}$ values

In order to assess the effect of adding more antennas at the BS, the spectral efficiency and fairness comparisons are repeated for $4 \times 1$ antenna configuration. As shown in Figure 4, evaluated algorithms have similar characteristics as in $2 \times 1$ case. It is observed that increasing 
the number of antennas at the BS has not any effect on the spectral efficiency in $m=1$ (Rayleigh channel) for max-SNR algorithm. However, employment of more antennas leads to slight increase of spectral efficiency in $m=3$ (weaker than Rayleigh channel), while that has an opposite effect in $m=0.6$ (severe than Rayleigh channel) case. Beyond that, the ORR algorithm gives more spectral efficiency for all values of the $m$ parameter in $4 \times 1$ antenna configuration. Finally, the spectral efficiency of RR algorithm is independent from the number of antennas.

Figure 5 shows the fairness performance of scheduling algorithms in $4 \times 1$ configuration. As seen from this plot, the fairness performance of max-SNR algorithm can be improved by using more antennas at the BS for all $m$ values. As predicted, the employment of more antennas at the BS does not vary the fairness index for RR and ORR algorithms.

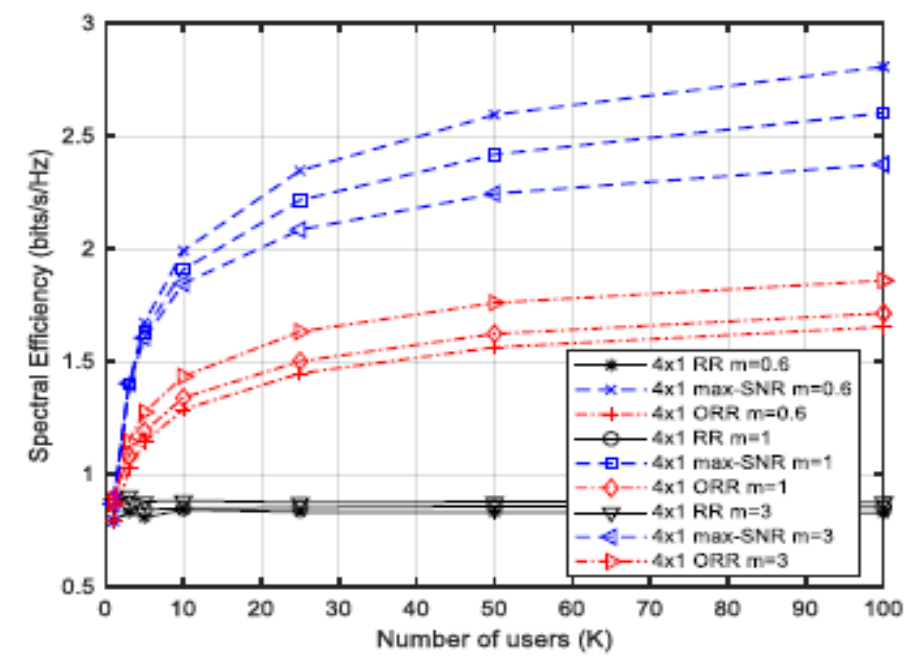

Figure 4. Spectral efficiency of scheduling algorithms in $4 \times 1$ antenna configuration for different $\mathrm{m}$ values

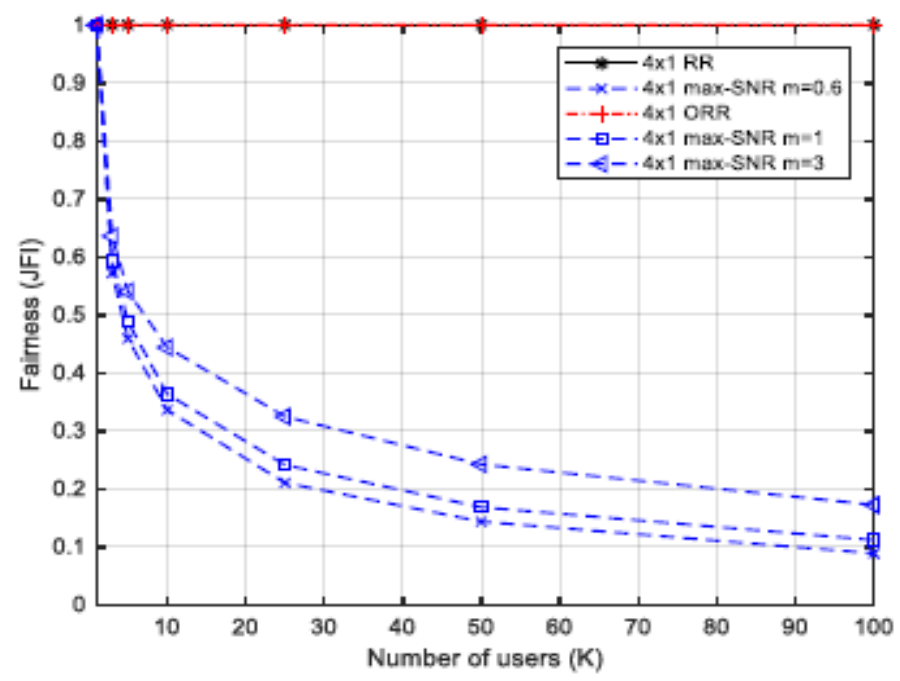

Figure 5. Fairness of scheduling algorithms in $4 \times 1$ antenna configuration for different $\mathrm{m}$ values 


\section{CONCLUSION}

This study presents a detailed performance analysis of well-known scheduling algorithms for cellular wireless networks in a Nakagami-m fading channel conditions. It was shown that the max-SNR scheduling algorithm provides a better spectral efficiency performance at the cost of unfairness among the users, while the RR and ORR algorithms guarantees the maximum user fairness at the cost of the performance loss in term of spectral efficiency. The Nakagami-m fading parameter has an effect on the spectral efficiency of max-SNR, ORR and RR algorithms. But, in max-SNR algorithm, this effect varies (positive/negative) depending on the $m$ parameter.

\section{REFERENCES}

[1] Zeng, M., Annamalai, A., Bhargava, V. K., (1999) "Recent advances in cellular wireless communications", in IEEE Communications Magazine, vol. 37, no. 9, pp. 128-138.

[2] Miao, G., Song, G., (2015) "Energy and Spectrum Efficient Wireless Network Design”, Cambridge University Press.

[3] Bölcskei, H., Gesbert, D., Papadias, C. B., Van Der Veen, A.-J., (2006) "Space-Time Wireless Systems: From Array Processing to MIMO Communications", Cambridge University Press.

[4] Rappaport, T., (2002), "Wireless Communications: Principles and Practice", Prentice Hall PTR.

[5] Wu, T.-Y., Huang, C.-Y., Chao, H.-C., (2003) "A survey of Mobile IP in cellular and Mobile Ad-Hoc Network environments", in Ad Hoc Networks, vol. 3, no. 3, pp. 351-370.

[6] Stallings, W., (2014) "Wireless Communications and Networks", Pearson Education Limited.

[7] Kyriazakos, S. A., Karetsos, G. T., (2004) "Practical Radio Resource Management in Wireless Systems", Artech House.

[8] Rasool, J., Hassel, V., de la Kethulle de Ryhove, S., Øien, G. E., (2011), "Opportunistic scheduling policies for improved throughput guarantees in wireless networks", vol. 43, 18 pages.

[9] Asadi A., Mancuso, V., (2013) "A Survey on Opportunistic Scheduling in Wireless Communications", in IEEE Communications Surveys \& Tutorials, vol. 15, no. 4, pp. 1671-1688.

[10] Liu, X., Chong, E. K. P., Shroff, N. B., (2003) "A framework for opportunistic scheduling in wireless networks" in Computer Networks, vol. 41, no. 4, pp. 451-474.

[11] Tse, D., Viswanath, P., (2005) "Fundamentals of Wireless Communication", Cambridge University Press.

[12] Knopp R., Humblet, P. A. (1995) "Information capacity and power control in single-cell multiuser communications", in Proc. IEEE Int. Conf. on Communications (ICC'95), vol. 1, pp. 331-335.

[13] Gyasi-Agyei, A. (2005) "Multiuser diversity based opportunistic scheduling for wireless data networks", in IEEE Communications Letters, vol. 9, no. 7, pp. 670-672.

[14] Pätzold, M., (2011) "Mobile Radio Channels", Wiley.

[15] Kumbhani, B., Kshetrimayum, R. S., (2017) "MIMO Wireless Communications over Generalized Fading Channels", CRC Press.

[16] Viswanath, P., Tse, D., Laroia, R., (2002) "Opportunistic beamforming using dumb antennas", in IEEE Transactions on Information Theory, vol. 48, no. 6, pp. 1277-1294.

[17] Gradshteyn, I. S., Ryzhik, I. M., (2007) "Table of Integrals, Series and Products", 7th ed. Academic Press, New York, 2007.

[18] Stefanovic, H., Savic, A., (2011) "Some general characteristics of Nakagami-m distribution", in Proceedings of International Symposium on Computing in Informatics and Mathematics (ISCIM 2011), pp. 695-705.

[19] Myers, A. D., Basagni, S., (2002) "Wireless media access control", in "Handbook of wireless networks and mobile computing", John Wiley \& Sons.

[20] Xia, F., Rahim, A., (2015) "MAC Protocols", in "MAC Protocols for Cyber-Physical Systems", Springer. 
[21] Hadded, M., Muhlethaler, P., Laouiti, A., Saidane, L., (2016) "A Centralized TDMA based Scheduling Algorithm for Real-Time Communications in Vehicular Ad Hoc Networks", in Proceedings of SoftCom 2016, Split, Croatia.

[22] Eltayeb, M. E., (2014) "Opportunistic Scheduling with Limited Feedback in Wireless Communication Networks", Dissertation, The University of Akron.

[23] Soy, H., Özdemir, Ö., (2016) "Fuzzy Logic Based Opportunistic Scheduler Design to Improve Fairness in Cellular Wireless Networks", International Journal of Modern Communication Technologies \& Research (IJMCTR), vol. 4, no. 9, 6 pages.

[24] Torabi, M., Haccoun, D., Ajib, W., (2009) "Multiuser Scheduling over MIMO Nakagami-m Fading Channels: Capacity and BER Performance" in Proceedings of IEEE 69th Vehicular Technology Conference, Barcelona, pp. 1-5.

[25] Kulkarni, S. S., Rosenberg, C., (2003) "Opportunistic scheduling policies for wireless systems with short term fairness constraints" in Proceedings of Global Telecommunications Conference, (GLOBECOM '03), pp. 533-537.

[26] Hassel, V., Hanssen, M. R., Oien, G. E., (2006) "Spectral Efficiency and Fairness for Opportunistic Round Robin Scheduling", in Proceedings of IEEE International Conference on Communications, Istanbul, pp. 784-789.

[27] Jain, R., Chiu, D., Hawe, W., (1984) “A quantitative measure of fairness and discrimination for resource allocation and shared computer systems", Digital Equipment Corporation (DEC) Research Report TR-301.

[28] Chang, C. H., Lee, Y., (2009) "Opportunistic beamforming systems with diversity combining", in Proceedings of 7 th International Conference on Information, Communications and Signal Processing (ICICS), pp. 1-5.

\section{AUTHOR}

Hakk1 Soy was born in Konya, Turkey in 1978. He received the B.S. degree in Electronics Engineering from Uludağ University, Bursa, Turkey in 1999 and the Ph.D. degree in Electrical and Electronics Engineering from Selçuk University, Konya, Turkey in 2013. He joined the Department of Electrical and Electronics Engineering, Necmettin Erbakan University, Konya, Turkey, where he is currently an Assistant Professor. His research interests include wireless sensor networks, sensoractuator networks, MAC protocols, scheduling algorithms, MIMO communication systems and opportunistic communication.

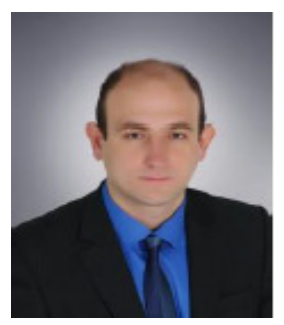

